changes that might precede an earthquake.

The plan was to leave those instruments in the hole to capture the many small repeating earthquakes that occur within a few hundred metres. For a few days, the devices worked well. But then they went quiet. William Ellsworth of the US Geological Survey in Menlo Park, California, another former principal investigator, says there may be an electrical short in one of the connections that carries data to the surface. Hot, corrosive groundwater in the hole could have damaged equipment.

Because the instruments are mounted on thick pipe, it would take a crane or a drill rig to pull the package back up. Right now, the project does not have the money to do that. In December, the team lowered one temporary

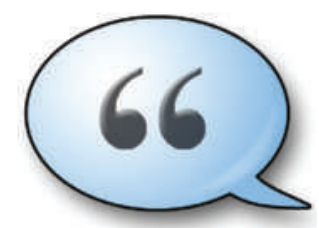

HAVE YOUR SAY

Comment on any of our News stories, online. www.nature.com/news

seismometer into the drill hole and is currently collecting data from that.

Ellsworth says some of the problems were discussed in December at a meeting of the American Geophysical Union in San Francisco. But there has been no general announcement to the public. "We were all embarrassed about it," says Zoback.

Richard Monastersky

\section{-}

\title{
Hubble: the last hurrah
}

On 11 May, the space shuttle Atlantis will head to the Hubble Space Telescope for a fifth and final servicing mission. With six batteries, six gyroscopes and a data interface system to be installed by astronauts, the telescope should be ready to perform for at least another five years. For scientists, the main feast will come with the new Wide Field Camera 3 and the Cosmic Origins Spectrograph, along with repairs to existing instruments. "I can hardly wait," says David Leckrone, senior project scientist for Hubble at NASA's Goddard Space Flight Center in Greenbelt, Maryland. Eric Hand looks at some of the most highly ranked proposals from outside scientists.
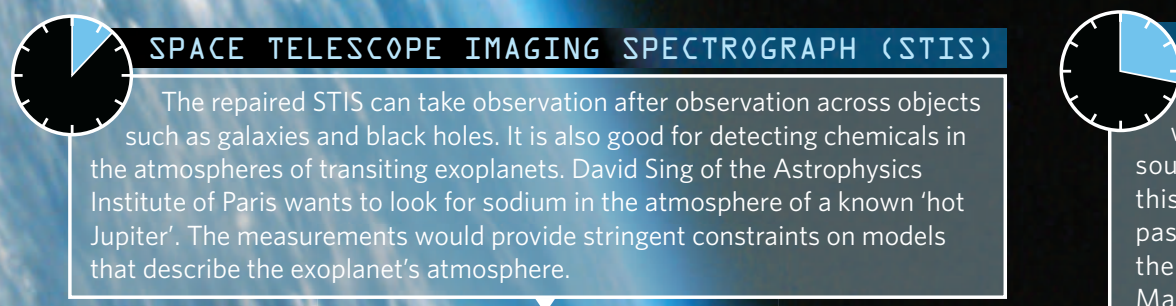

COSMIC ORIGINS SPECTROGRAPH (COS)

COS will be very sensitive to far ultraviolet light and well suited to splitting up the light created by point sources, such as quasars and stars. Absorption features in this light reveal details of the clouds of gas the light has passed through. At the moment, astronomers can't find al the gas that they expect. Todd Tripp of the University of Massachusetts in Amherst will use COS to look for cool

gas clouds backlit by quasars.

NICMOS, an actively cooled instrument in the near infrared, has been offline since September, and several reboot attempts haven't worked. Engineers suspect that an ice particle in its cryocooler could be causing problems. time spent on each instrument.

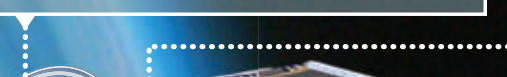

